Methodology A 32-item questionnaire addressing multiple issues related to the SUCCOR study and surgical management of ECC was designed and sent from the ESGO account through a newsletter on February 23rd to all ESGO members from 54 countries of the ESGO directory. The survey was completely confidentially and could be completed in less than 5 minutes. Responses from 353 members were obtained. Descriptive statistical analysis and frequency tables, as well as two-sided Fisher's exact test, were used.

Result(s)* $92.9 \%$ of responders were aware of the findings of the SUCCOR study. $73.4 \%$ expected the results obtained. 5 out of 10 of members confirmed that have changed their practice after the publication of the manuscript.

Currently, $34.8 \%$ of responders admit that they usually perform MIS (laparoscopy/robotic) for cervical tumours smaller than $2 \mathrm{~cm}$, while for tumours larger than $2 \mathrm{~cm}$ (FIGO IB2) $15.1 \%$ state that they continue to carry out MIS. Between 63 and $64.1 \%$ apply protective maneuvers during this procedure. In ECC who have undergone conization, 56.1\% would performed MIS for tumours smaller than $2 \mathrm{~cm}$ while only $30 \%$ would offer it for tumours larger than $2 \mathrm{~cm}$.

Finally, $84.1 \%$ of ESGO members consider ethically acceptable the development of a new European prospective observational study (Image 1) to evaluate the surgical approach in ECC (IB1-IB2).

Conclusion* According to the ESGO members' perspectives regarding the approach to ECC, it seems that MIS still has an important role to play, which should be evaluated in a new prospective study that reflects the European reality (Image 2).

\section{INCIDENCE OF CERVICAL CANCER AND MANAGEMENT OF HIGH GRADE SMEARS IN WOMEN AGED 50 YEARS AND ABOVE}

R Shah*, E Leung, D Mukhopadhyay. Queen's Hospital, UK

\subsection{6/ijgc-2021-ESG0.69}

Introduction/Background* Interpretation of smears is a challenge in older women owing to atrophic changes. Colposcopy can be difficult and views are repeatedly unsatisfactory due to a stenotic os. LLETZ procedures are therefore often performed based on initial high-grade smear cytology. However, approximately $60 \%$ of cervical cancers occur in women aged 45 plus; evidence shows a second peak of high-risk HPV in postmenopausal women. This study aims to review the management of women aged 50 years and above with high-grade smears and establish the incidence of cervical cancer in this cohort.

Methodology A retrospective study was conducted; an electronic search was undertaken to identify all women aged 50 years and above referred from the national screening programme with a high grade (moderate or severely dyskaryotic) smear to the Colposcopy unit at Queen's Hospital \& King George Hospital Barking, Havering \& Redbridge NHS Trust between $1^{\text {st }}$ January $2016-31^{\text {st }}$ December 2019. Infoflex and cyberlab were used to collate data to establish establish colposcopy findings, histology of biopsy, LLETZ, or further surgical intervention plus results following tests of cure. Data was analysed using Microsoft Excel.

Result(s)* Of the 99 women referred, smear cytology demonstrated 1 suspicious of glandular neoplasia, 50 of severe dyskaryosis and 48 of moderate dyskaryosis. 11 patients were excluded due to incomplete data. Colposcopic views were undiagnostic for 27 (31\%) of patients. 82 (93\%) patients underwent LLETZ, while $11 \%$ of patients underwent radical surgery based on their smear +/- histology results. This study identified a 5\% incidence of cancer (3 SCC; 1 adenocarcinoma). $61 \%$ exhibited high-grade histology (23\% CINII and $38 \%$ CINIII) and 13\% exhibited low-grade histology (CINI). $11 \%$ had no abnormality and a further $10 \%$ displayed other benign changes.

Conclusion* Our local analyses demonstrate a 5\% incidence of cancer in women aged 50 years as per histological diagnosis. $61 \%$ of referred patients exhibited high-grade histology; $11 \%$ of the sample population underwent radical surgery. This may justify a lower threshold to performing LLETZ procedures where colposcopy findings are indeterminate.

\section{EARLY OUTCOMES OF NEOADJUVANT CHEMOTHERAPY AND SUBSEQUENT RADIATION THERAPY IN NON METASTATIC ADVANCED CERVICAL CANCER}

${ }^{1} \mathrm{R}$ Mittal, ${ }^{1} \mathrm{~L}$ Gurram, ${ }^{1} \mathrm{C}$ Johny, ${ }^{2} \mathrm{~S}$ Gupta, ${ }^{2} \mathrm{~J}$ Ghosh, ${ }^{2} \mathrm{~S}$ Gulia, ${ }^{1} \mathrm{~S}$ Chopra, ${ }^{1} \mathrm{R}$ Engineer, ${ }^{3} \mathrm{ST} \mathrm{S},{ }^{3} \mathrm{~A}$ Maheshwari, ${ }^{2} \mathrm{~S}$ Rath, ${ }^{3} \mathrm{P}$ Poddar, ${ }^{4} \mathrm{P}$ Popat, ${ }^{1} \mathrm{D}$ A, ${ }^{4} \mathrm{U}$ Mahantshetty. ${ }^{1}$ TATA MEMORIAL HOSPITAL, Homi Bhabha Block, Radiation Oncology, Mumbai, India; ${ }^{2} T A T A$ MEMORIAL HOSPITAL, Homi Bhabha Block, Medical Oncology, Mumbai, India; ${ }^{3}$ TATA MEMORIAL HOSPITAL, Homi Bhabha Block, Surgical Oncology, Mumbai, India; ${ }^{4}$ TATA MEMORIAL HOSPITAL, Homi Bhabha Block, Radiodiagnosis, Mumbai, India

\subsection{6/ijgc-2021-ESG0.70}

Introduction/Background* To report outcomes of non metastatic advanced cervical cancer patients treated with neoadjuvant chemotherapy (NACT) followed by chemoradiation/radiation. Methodology 104 patients treated between January 2015 and December 2019 were analyzed and restaged as per FIGO 2018. RECIST criteria v1.1 was used to assess response to NACT and toxicity was assessed using CTCAE v4.0. Survival analysis was done using Kaplan-Meir method. Univariate and multivariate analysis was done using Log-rank test and Coxregression model respectively.

Result(s)* 104 patients (median age: 52 years) of advanced cervical cancer (92\% SCC) received NACT for following reasons: bulky pelvic nodes $(n=20)$, bulky para-aortic nodes $(n=10)$, bulky pelvic + para-aortic nodes $(n=31)$, bladder invasion $(n=43)$. Baseline median pelvic and para-aortic nodal size was $2.5 \mathrm{~cm}$ (range: 1-8) and $1.8 \mathrm{~cm}$ (range: 13.5) respectively. Baseline median tumor infiltration size into bladder on cystoscopy was $3 \mathrm{~cm}$ (range 0.5-7). NACT regimen was Paclitaxel $(175 \mathrm{mg} / \mathrm{m} 2)$ and Carboplatin (AUC 5) 3 weekly IV infusion for 3 (median) cycles (range: 1-6). Post NACT completion, 9 (8.6\%) defaulted from subsequent treatment, $8(7.7 \%)$ were labeled as best supportive care, 1 received palliative chemotherapy for progressive disease and $13(12.5 \%)$ patients received palliative RT. 73 (70.2\%) patients received radical pelvic EBRT (median dose of $46 \mathrm{~Gy} / 23 \mathrm{Fr})$ of which $68(93.1 \%)$ received brachytherapy boost (median dose 21Gy/3Fr). 32 (43.8\%) and 28 (38.3\%) patients also received extended field RT (EFRT) and nodal boost (median dose 5.4Gy/3Fr) respectively. 64 (87.6\%) patients received concurrent cisplatin. On univariate analysis, patients with comorbidities, bladder invasion, pelvic nodal disease, NACT dose reduction, incomplete radical treatment post-NACT and pelvic nodal size $\geq 2.5 \mathrm{~cm}$ at baseline had poor outcomes (table 1). On multivariate 